\title{
Sugar, Cavities, Systemic Disease and Addiction?
}

\begin{abstract}
Historically and clinically, sugar has been shown to be a major causative factor in dental caries. Excess sugar intake in children has shown to be related to children developing less than $80 \%$ of their idea weight, lower vitamin D levels, lower calcium levels, lower albumin concentrations, lower ferritin status, greater odds for iron deficiency anemia and children with higher levels of untreated carieswere found to have poorergrowth and elevated BMI percentiles, which increased risk to general health. Therefore at an early age, sugar has caused systemic and dental effects. These effects get more pronounced in adulthood, where sugar is shown to be related to cardiovascular disease, including high blood pressure and stroke, diabetes type 2 , fatty liver, cancerincluding breast cancerand metastasis, additionally fructose is related to pancreatic cancer.

Asmembers of health care team, it is our professional responsibility to note the general health of patients and to make appropriate referrals to other health care professionals and to know how the diseases/disorders of the patient will impact on the dental treatment. When multiple dental caries are found and high sugar intake is suspected, appropriate referrals are important to the overall health of the patient
\end{abstract}

\section{Introduction}

\section{Before mankind was introduced to sugar}

Mankind was introduced to dental caries and degenerative diseases approximately $10,000 \mathrm{BC}$, when the diet changed as a result of moving from a hunter-gatherer to the agriculture life style. The same phenomena occurred in more recent times when primitive people on primitive diets and basically free of dental caries and degenerative diseases were introduced to the modern diet. Additionally current data, which was generated by the US government in the NHANES study, show that dental caries in children precede degenerative diseases later in life. Other studies are finding some degenerative changes in adolescence. Thus, diet and dental caries should be given greater importance as they can be an indicator of the probability of future degenerative diseases [1].

Caselitz analyzed the historical evolution of caries in 518 human populations of Europe, Asia and America in a wide timeline from the Paleolithic to the present, confirming that during Paleolithic and Mesolithic periods, the hunter-gatherers had less caries and lesions progressed more slowly $[2,3]$. Caries indices have increased gradually from Neolithic times, until they reach the high rates observed at the present. Remarkable caries increments occurred during the second half of the 19th century, when dramatic increases in the intake of sugar and refined carbohydrates between 1830 and 1880 [2-4].

Primitive people in modern times with no caries and systemic health

Price observed primitive people on natural diets and found that less than $1 \%$ of these people had decay in their permanent teeth.

\section{Journal of} Oral Biology

\section{George E. White*}

Pediatric Dentistry, Nova Southeastern University, Health Professions Division, College of Dental Medicine,

Post Graduate Pediatric Clinic, 819 East 26th Street, Fort Lauderdale, FL 33305, USA

\section{*Address for Correspondence}

George E. White, Pediatric Dentistry, Nova Southeastern University, Health Professions Division, College of Dental Medicine, Post Graduate Pediatric Clinic, 819 East 26th Street, Fort Lauderdale, FL 33305, USA, Tel: 954-567-5650; Fax: 877-991-4957; E-mail: gwhite1@nova.edu

Submission: 20 June, 2016

Accepted: 1 July, 2016

Published: 5 July, 2016

Copyright: ๑ 2016 George EW, et al. This is an open access article distributed under the Creative Commons Attribution License, which permits unrestricted use, distribution, and reproduction in any medium, provided the original work is properly cited.

He found that freedom from caries always went with freedom from chronic diseases like cancer and heart disease and infectious diseases like tuberculosis, which at the time was a world-wide epidemic [5]. Others have reported the lack of degenerative diseases, particularly cancer in isolated groups [6-9].

More importantly, these people in Price's study were healthy with well formed, broad faces and teeth in perfect alignment. Their bodies were wonderful in physical development [5]. The diets of the healthy "primitives" Price studied were all very different (in other words it was not so much what they ate that contributed to their health as what they did not eat).

Similar findings have been found recently in several populations living in isolated areas of the world where they kept their ancestral ways of life (e.g. many African tribes, Inuits, South American Indians, Melanesian, Polynesian, under conditions of perfect adaptation to their environments and diets [7-9].

Lanfranco and Eggers reviewed many studies on the frequency of caries in prehistoric man and summarized the findings as follows: hunter-gathers had a caries frequency ranging from 0-5.3; fisherman 0.4-10.3 and farmers 2.2-26.9 [10]. While there are disputes among investigators as to the method of gathering data, the trend is clear, as the diet changed, the amount of dental caries increased.

Of great importance for the hunter-gather, they rarely died of coronary artery disease, diabetes mellitus or chronic obstructive pulmonary disease, among other ailments common in societies like ours. They may not have been healthier in absolute terms, but they were absent infection and osteoarthritis and rarely had chronic diseases that we commonly have [10-12]. Therefore, low caries (low sugar intake) and lack of degenerative diseases are found together in population studies.

\section{High Sugar Intake, Caries and Systemic Disease Children}

When questioning the impact of dental health on quality of life, 
Alsumait et al. studied at total of 440 children aged 11-12 years old. They concluded that the increase in the number of carious teeth was associated with a limitation in oral functions and that preventive treatment had a positive impact on emotional well-being and restorative treatments improved oral function [12].

Nutrition affects dental health and general health. Children with early childhood caries (ECC) were significantly more likely to weigh less than $80 \%$ of their ideal weight, thereby satisfying one of the diagnostic criteria for failure to thrive [13].

Similar findings were more recently found that suggests that higher levels of untreated caries are associated with poorer oral functions and overall growth in school children [12-14].

Young children with ECC or S-ECC (severe-childhood caries) were very likely to be found with systemic effects showing how this spectrum of health degeneration begins. They were likely:

${ }^{*}$ Weigh less than $80 \%$ of their ideal weight [12]

${ }^{\star}$ Low in vitamin D [15]

${ }^{\star}$ Low in Calcium [15]

${ }^{*}$ Low in albumin concentrations [15]

${ }^{\star}$ Low ferritin status [15]

${ }^{*}$ Greater odds for iron deficiency anemia [16]

Children with higher levels of untreated caries:

${ }^{*}$ poorer growth $[13,14]$

${ }^{\star}$ Elevated BMI percentiles increased risk to general [17].

Often excess sugar is given to young children as a means of comforting a child and as a cheap food source, which results in malnourishment.

Malnourished children have been shown to have decrease physical activity and endurance, poorer cognitive function and school performance. Multiple single micronutrient deficiencies, including vitamin $\mathrm{B} 12$, thiamin, niacin, zinc and iron, have been associated with poorer cognitive performance. Behavioral problems, including attention deficits, have also been associated with food insufficiency and malnutrition.

\section{Adults}

When examining adults for caries experience and overall health status, Johnston and Vieira found the following diseases and conditions associated with higher caries experience: hepatitis, asthma, high blood pressure, stroke, liver disease and diabetes [18].

\section{High sugar intake increases risk for cardiovascular disease}

Diets high in sugar may induce increased risk of coronary heart disease, have elevated levels of triglycerides, glucose, insulin and uric acid, impaired glucose tolerance, insulin and leptin resistance, nonalcoholic fatty liver disease, and altered platelet function. A diet high in added sugars has been found to cause a 3 fold increased risk of death due to cardiovascular disease.

Sugars are diverse class of compounds. The monosaccharide fructose and fructose-containing sweeteners (e.g. sucrose) produce greater degrees of metabolic abnormalities than does glucose (either isolated as a monomer, or in chains as starch) and may present greater risk of CHD. To reduce the burden of CHD, guidelines should focus particularly on reducing intake of concentrated sugars, specifically the fructose-containing sugars like sucrose and high-fructose corn syrup in the form of ultra-processed foods and beverages [19] Table 1.

Table 1: Avoid These Foods with Highly Processed Sugar.

1. Sugars, Syrups and Sweeteners - up to $100 \%$ sugar

2. Drink Powders and Soft Drinks - up to $95 \%$ sugar

3. Candies and Nougat - up to $93 \%$ sugar

4. Dried Fruit - up to $61 \%$ sugar

5. Cookies, Cakes and Pies - up to $71 \%$ sugar

6. Jams, Preserves and Spreads - up to $60 \%$ sugar

7. Cereals (Kellogg's Honey Snacks, etc.) - up to $56 \%$ sugar

8. Fruit Canned in Syrup - up to $52 \%$ sugar

9. Sauces and Instant Gravies - up to $38 \%$ sugar

10. Ice Cream, Frozen Yogurt and Milkshakes - up to 25\% sugar

Others have found that consumption of added sugar is associated with development and/or prevalence of fatty liver, dyslipidemia, insulin resistance, hyperuricemia, CVD and type 2 diabetes and failure to stimulate leptin production [20-23].

\section{High sugar intake increases risk of insulin resistance}

Since diets high in sugar can result in insulin resistance, it is significant that chronic hyperinsulinemia from insulin resistance is involved in the etiology of endometrial cancer [24].

In counseling patients to reduce the risk of cardiovascular disease risk, it is advised to restrict sugar intake as excess sugar intake is more likely than traditional factors to lead to atherosclerotic disease [25].

\section{High sugar intake increases risk for cancer}

Similar restrictions to sugar intake should be given to cancer patients. Granja et al. discuss cancer cells aerobic metabolism and glucose. While normal differentiated cells primarily use mitochondrial respiration to generate the required energy for cellular processes, most cancer cells rely on glycolysis, even in sufficient oxygen conditions. This phenomenon is known as the "Warburg effect" or aerobic glycolysis and the metabolic reprogramming of cancer cells towards this altered energy metabolism is currently recognized as one of the "hallmarks of cancer". Aerobic glycolysis underlies the rapid growth of tumor cells, with high rates of glucose consumption and lactic acid production, leading to cellular acidosis [26].

Similar comments concerning the metabolism of cancer cells and glycolysis were made by Elf and Chen [27].

Using cancer metabolism as a therapeutic target, namely aerobic glycolysis in lieu of oxidative phosphorylation, were also discussed by Batra et al. [28]. 
What is the association between health related quality of life and the development of dental caries in adults? Akesson et al. studied over 9,838 individuals over a 2 to 7 year period. They found that caries increment increased significantly with decreasing scores for both physical and mental dimensions in women but no associations in men. However, lifelong caries experience (DMFS) increased linearly with decreasing physical health related quality of life in both men and women; this was also observed for the single dimension of mental health related quality of life. One of the many confounders was sugar intake [29].

\section{High sugar intake increases risk for breast cancer and metastasis}

Recently, animal studies performed at The University of Texas MD Anderson Cancer Center and funded in part by the National Institutes of Health showed that high amounts of dietary sugar increase the risk of breast cancer and metastasis to the lungs. These researchers determined that fructose derived from the sucrose was responsible for facilitating lung metastasis and 12-HETE production in breast tumors. Overall, these data suggested that dietary sugar induces 12-LOX signaling to increase risks of breast cancer development and metastasis [30].

\section{High fructose intake increases risk for pancreatic cancer}

Research conducted at the University of California in Los Angeles (UCLA) found that pancreatic tumor cells use fructose to divide and proliferate. Traditionally, glucose and fructose have been considered as interchangeable monosaccharide substrates that are similarly metabolized and little attention has been given to sugars other than glucose. However, fructose intake has increased dramatically in recent decades and cellular uptake of glucose and fructose uses distinct transporters. Liu et al. report that fructose provides an alternative substrate to induce pancreatic cancer cell proliferation. Importantly, fructose induces thiamine-dependent transketolase flux and is preferentially metabolized via the nonoxidative pentose phosphate pathway to synthesize nucleic acids and increase uric acid production. These findings show that cancer cells can readily metabolize fructose to increase proliferation. They have major significance for cancer patients given dietary refined fructose consumption, and indicate that efforts to reduce refined fructose intake or inhibit fructose-mediated actions may disrupt cancer growth [31].

Whereas, glucose favors overall growth kinetics, fructose enhances protein synthesis and appears to promote a more aggressive cancer phenotype. Fructose intake is associated with increased risk of pancreatic and small intestinal cancers and possibly others. Fructose promotes flux through the pentose phosphate, which enhances protein synthesis and may directly increase tumor growth, is associated with more aggressive cancer behavior and may promote metastasis [32].

If sugar is related to poor oral and general health, why do people continue to ingest it?

\section{Is sugar addiction real?}

Animal studies find that sugar is more rewarding and addictive than cocaine $[33,34]$. Interestingly, whereas drugs are thought to be very highly reinforcing, rodents are more likely to work for sweet rewards such as sucrose or saccharin, even when not food deprived, than they will for cocaine. Although extended access to cocaine increases the reinforcing efficacy of the drug much more than for sweet tastants, rodents are still more likely to work for sucrose or saccharin after chronic exposure to cocaine [35].

It appears that humans are neurologically programmed to seek sugar. Of course the basic metabolism of humans is based on glucose.

Eating too much sugar for too long results in re-programming the neurological circuits of the brain similar to the brain of a heroin or cocaine addict. This is caused by a surge of dopamine, which increases the desire for the taste of sweet [36].

To continue with the comparison, sugar withdrawal is similar to heroin withdrawal, which includes anxiety, depression followed by cravings [37].

Schulte et al. concluded that preliminary evidence shows that not all foods are equally implicated in addictive-like eating behavior and highly processed foods, which may share characteristics with drugs of abuse (e.g. high dose, rapid rate of absorption) appear to be particularly associated with "food addiction" [38].

Researchers vary on the topic of whether food "addiction" is the same as drug addiction, but no matter the outcome, it is a major health problem [39].

\section{Smoking as a 50 Year Public Health Problem}

Smoking tobacco has been shown to have systemic and periodontal effects. For some it is an addiction and difficult to stop using tobacco. Interestingly, it will be shown that it raises blood glucose. Smoking is discussed here because while tobacco was known for a long time to cause diseases, it took decades to educate the public to reduce smoking in the U.S. Currently it is known that excess sugar causes diseases, but the public has not been informed enough to reduce or eliminate it from their diet.

For the United States, the epidemic of smoking caused disease in the twentieth century ranks among the greatest public health catastrophes of the century, while the decline of smoking consequent to tobacco control is surely one of public health's greatest successes. However, the current rate of progress in tobacco control is not fast enough, and much more needs to be done to end the tobacco epidemic. Unacceptably high levels of smoking attributable disease and death, and the associated costs, will persist for decades without changes in our approach to slowing and even ending the epidemic. If smoking persists at the current rate among young adults in this country, 5.6 million of today's Americans younger than 18 years of age are projected to die prematurely from a smoking-related illness (Chapter 12). More than 20 million Americans have died as a result of smoking since the first Surgeon General's report on smoking and health was released in 1964 . Most were adults with a history of smoking, but nearly 2.5 million were nonsmokers, who died from heart disease or lung cancer caused by exposure to secondhand smoke. Another 100,000 were babies, who died of sudden infant death syndrome (often referred to as SIDS) or complications from prematurity, low birth weight, or other conditions caused by parental smoking, particularly smoking by the mother Table 2 [40]. 
Table 2: Premature deaths caused by smoking and exposure to secondhand smoke, 1965-2014.

\begin{tabular}{|c|c|}
\hline Cause of death & Total \\
\hline $\begin{array}{c}\text { Smoking-related cancers } \\
\text { Cardiovascular and metabolic } \\
\text { diseases }\end{array}$ & $6,587,000$ \\
\hline Pulmonary diseases & $7,787,000$ \\
\hline $\begin{array}{c}\text { Conditions related to pregnancy and } \\
\text { birth }\end{array}$ & $3,804,000$ \\
\hline $\begin{array}{c}\text { Lung cancers caused by exposure to } \\
\text { secondhand smoke }\end{array}$ & 108,000 \\
\hline $\begin{array}{c}\text { Coronary heart disease caused by } \\
\text { exposure to secondhand smoke }\end{array}$ & 263,000 \\
\hline Total & $2,194,000$ \\
\hline
\end{tabular}

Source: Centers for Disease Control and Prevention, National Center fo Chronic Disease Prevention and Health Promotion, Office on Smoking and Health, unpublished data.

As these figures illustrate, the harms caused by the historic patterns of tobacco use in the United States, and especially by cigarette smoking, are staggering. More than 10 times as many U.S. citizens have died prematurely from cigarette smoking than have died in all the wars fought by the United States during its history. Study after study has confirmed the magnitude of the harm caused to the human body by exposure to toxicants and carcinogens found in tobacco smoke. Since 1964, the 31 previous Surgeon General's reports have chronicled a still growing but already conclusive body of evidence about the adverse impact of tobacco use on human cells and organs and on overall health. Health statistics show that all populations are affected [40].

Marta and Lopez-Jornet found that smoking increased glucose and low density lipoproteins in the blood and are associated with a risk of oral leukoplakia [41]. This another interesting link of sugar to general health.

The central theme of this article is that the oral conditions can reflect what is happening to the body. Smoking is a chemical link that shows its effects in the oral cavity and the general body. Periodontal disease also has been shown to affect tissues distant to the oral cavity via microbes. The use of excess sugar, which is a chemical, likewise will affect oral health and the body.

\section{Summary}

Just as national health organizations such as CDC, American Dental Association and American Medical Association and national legislative organizations worked to eliminate smoking from our society because of the overall detrimental effects that it has on oral tissues and systemic tissues, the same should be done for excess sugar in the diet as it is related to detrimental effects on oral tissues and systemic tissues.

\section{References}

1. White GE (2015) Diet, dental caries and other degenerative diseases. J Oral Biol 2: 6.

2. Caselitz P (1998) Caries ancient plague of humankind. In: Alt KW, Rosing FW, Teschler-Nicola M (Eds). Dental anthropology: fundamentals, limits and prospects. Springer Wien New York, pp. 203-226.
3. Hu FB (2013) Resolved: there is sufficient scientific evidence that decreasing sugar-sweetened beverage consumption will reduce the prevalence of obesity and obesity-related diseases. Obes Rev 14: 606-619.

4. Moore WJ, Corbett ME (1975) Distribution of dental caries in ancient British populations. III. The 17th century. Caries Res 9: 163-175.

5. Price WA (1945) Nutrition and physical degeneration. Price-Pottenger Nutrition Foundation, San Diego, CA.

6. Richards MP (2002) A brief review of the archeological evidence for paleolithic and neolithic subsistence. Eur J Clin Nutr 56: 16 p following 1262.

7. Mayhall JT (1970) The effect of culture change upon the Eskimo dentition. Artic Anthropol 7: 117-121.

8. Shuler CF (2001) Inherited risks for susceptibility to dental caries. J Dent Educ 65: 1038-1045.

9. Donnelly CJ, Thomson LA, Stiles HM, Brewer C, Neel JV, et al. (1997) Plaque, caries, periodontal diseases and accumulation among Yanomamö Indians, Venezuela. Community Dent Oral Epidemiol 5: 30-39.

10. Lanfranco LP, Eggers S (2012) Caries through time: an anthropological overview. In: Li MY (Ed). Contemporary Approach to Dental Caries. InTech.

11. Molnar S, Molnar I (1985) Observations of dental diseases among prehistoric populations of Hungary. Am J Phys Anthropol 67: 51-63.

12. Alsumait A, EISalhy M, Raine K, Cor K, Gokiert R, et al. (2015) Impact of dental health on children's oral health-related quality of life: a cross-sectional study. Health Qual Life Outcomes 13: 98.

13. Sanchez A, Reeser JL, Lau HS, Yahiku PY, Willard RE, et al. (1973) Role of sugars in human neutrophilic phagocytosis. Am J Clin Nutr 26: 1180-1184.

14. Smith R (1998) Vegetarian studies. Health and healing wisdom. PricePottenger Nutrition Foundation, Winter 30: 574-776.

15. Kabara JJ (1989) The pharmacological effects of lipids. In: Kabara JJ (Ed) The American Oil Chemists Society, pp. 366.

16. Ros OR et al, (1998) AOCS Proceedings: In: Garg ML (Ed.). The FASEB Journal, Chicago, IL, pp. 7.

17. Lawson LD, Kummerow FA (1979) $\beta$-oxidation of the coenzyme A esters of vaccenic, elaidic and petroselaidic acids by rat heart mitochondria. Lipids 14 : 501-503.

18. Gagne G (1993) Mouth diseases in prehistoric agricultural population of northeastern North America. J Can Dent Assoc 59: 686-692.

19. Johnston L, Vieira AR (2014) Caries experience and overall health status. Oral Health Prev Dent 12: 163-170.

20. DiNicolantonio JJ, Lucan SC, O'Keefe JH (2016) The evidence for saturated fat and for sugar related to coronary heart disease. Prog Cardiovasc Dis 58: 464-472.

21. Stanhope KL (2016) Sugar consumption, metabolic disease and obesity: the state of the controversy. Crit Rev Clin Lab Sci 53: 52-67.

22. Dornas WC, de Lima WG, Pedrosa ML, Silva ME (2015) Health implications of high-fructose intake and current research. Adv Nutr 6: 729-737.

23. Malik VS, Pan A, Willett WC, Hu FB (2013) Sugar-sweetened beverages and weight gain in children and adults: a systematic review and meta-analysis. Am J Clin Nutr 98: 1084-1102.

24. Allman-Farinelli M, Partridge SR, Roy R (2016) Weight-related dietary behaviors in young adults. Curr Obes Rep 5: 23-29.

25. Hernandez AV, Pasupuleti V, Benites-Zapata VA, Thota P, Deshpande A, et al. (2015) Insulin resistance and endometrial cancer risk: a systematic review and meta-analysis. Eur J Cancer 51: 2747-2758.

26. Thornley S, Tayler R, Sikaris K (2012) Sugar restriction: the evidence for a drug-free intervention to reduce cardiovascular disease risk. Intern Med J 42 Suppl 5: 46-58.

27. Granja S, Pinheiro C, Reis RM, Martinho O, Baltazar F (2015) Glucose 
addiction in cancer therapy: advances and drawbacks. Curr Drug Metab 16 221-242.

28. Elf SE, Chen J (2014) Targeting glucose metabolism in patients with cancer Cancer 120: 774-780

29. Batra S, Adekola KU, Rosen ST, Shanmugam M (2013) Cancer metabolism as a therapeutic target. Oncology (William Park) 27: 460-467.

30. Åkesson ML, Wärnberg Gerdin E, Söderström U, Lindahl B, Johansson I (2016) Health-related quality of life and prospective caries development BMC Oral Health 16: 15.

31. Jiang Y, Pan Y, Rhea PR, Tan L, Gagea M, et al. (2016) A sucroseenriched diet promotes tumorigenesis in mammary gland in part through the 12-lipoxygenase pathway. Cancer Res 76: 24-29.

32. Liu H, Huang D, McArthur DL, Boros LG, Nissen N, et al. (2010) Fructose induces transketolase flux to promote pancreatic cancer. Cancer Res 70 : 6368-6376.

33. Port AM, Ruth MR, Istfan NW (2012) Fructose consumption and cancer: is there a connection? Curr Opin Endocrinol Diabetes Obes 19: 367-374.

34. Lenoir M, Serre F, Cantin L, Ahmed SH (2007) Intense sweetness surpasses cocaine reward. PLoS One 2: e698.

35. Ahmed SH, Avena NM, Berridge KC, Gearhardt AN, Guillem K (2013) Food addiction. In: Neuroscience in the $21^{\text {st }}$ Century. Springer New York, pp. 28332857.
36. DiLeone RJ, Taylor JR, Picciotto MR (2012) The drive to eat: comparisons and distinctions between mechanisms of food reward and drug addiction. Nat Neurosci 15: 1330-1335.

37. Tang DW, Fellows LK, Small DM, Dagher A (2012) Food and drug cues activate similar brain regions: a meta-analysis of functional MRI studies. Physiol Behav 106: 317-324.

38. Avena, NM, Rada P, Hoebel BG (2008) Evidence for sugar addiction: behavioral and neurochemical effects of intermittent, excessive sugar intake. Neurosci Biobeha Rev 32: 20-39.

39. Schulte EM, Avena NM, Gearhardt AN (2015) Which foods may be addictive? The roles of processing, fat content, and glycemic load. PLoS One 10: e0117959.

40. CDC (2014) 2014 Surgeon General's Report: The Health Consequences of Smoking-50 Years of Progress. U.S. Department of Health and Human Services. U.S. Department of Health and Human Services, Centers for Disease Control and Prevention, National Center for Chronic Disease Prevention and Health Promotion, Office on Smoking and Health, Atlanta.

41. Marta GF, Lopez-Jornet P (2016) Association between smoking, glycemia blood lipoproteins, and risk of oral leukoplakia. Aust Dent J [Epub ahead of print]. 